\title{
Crossing the boundaries
}

\author{
Heather B Wood
}

Specialization is an important concept in clinical medicine, and it has advantages and disadvantages. For the patient, there are obvious benefits in being able to consult a physician who has in-depth knowledge and extensive experience of their condition. If clinicians become too specialized, however, they run the risk of creating artificial boundaries that are not necessarily respected by the disease in which they specialize, and also risk focusing more on the individual disorder than on the whole patient.

The Therapy Insight reviews in Nature Clinical Practice Neurology highlight the importance of looking beyond the initial disease presentation when considering treatment options. These articles have a specific purpose-namely, to discuss medical conditions being managed by physicians in other specialties that have particular relevance to neurology, and to consider how these ostensibly non-neurological medical conditions could influence treatment decisions and patient management by neurologists. In previous issues, for example, we have considered the impact of type 1 and type 2 diabetes on brain development and function.

In a Therapy Insight in this issue, Alison Pack discusses the best strategies for managing epilepsy during pregnancy. This is an important subject to address, because both seizures and antiepileptic medications have the potential to cause adverse effects in the unborn child. Pregnancy is accompanied by dynamic physiological changes that can affect both seizure frequency and the metabolism of antiepileptic drugs, so preconceptual counseling, and close monitoring of the mother and fetus
The emergence of new clinical subspecialties might be perceived as a divisive process, but this need not be the case

HB Wood is the Editor of Nature Clinical Practice Neurology.

\section{Competing interests}

The author declared she has no competing interests.

www.nature.com/clinicalpractice doi:10.1038/ncpneuro0152 during pregnancy, are vital. According to Pack, the ideal person to monitor a pregnant woman with epilepsy would be an obstetrician who is also familiar with epilepsy and its treatment.

The emergence of new clinical subspecialties might be perceived as a divisive process, but this need not be the case. In fact, it can lead to unexpected collaborations between experts from seemingly disparate specialties, a prime example being the merging of the neurology and immunology fields to create the relatively new discipline of neuroimmunology. Traditionally, neurology and immunology were considered to share little common ground, as the CNS was believed to be largely exempt from immunological surveillance. It is now becoming increasingly clear, however, that the immune system has crucial roles in brain health and disease, and that the etiologies of many common neurological disorders, including multiple sclerosis (MS), Alzheimer's disease and stroke, have a strong immunological component.

The immunopathogenesis of MS is the topic of a review in this issue by Bernhard Hemmer and colleagues. They discuss how various immune cells and molecules are thought to participate in various stages of the MS disease process, and consider how these factors might be targeted by immunomodulatory therapies, some of which are already in use in the clinic.

These articles illustrate the value of employing a multidisciplinary approach when devising strategies for managing patients with neurological disorders or other conditions that are likely to have an impact on the nervous system. 